\title{
Drug Compliance in Children with Juvenile Idiopathic Arthritis and Reasons for Poor Compliance
}

\author{
Mohammad Humayun Kabir, Kamrul Laila*, Mujammel Haque, Shahana Akhter Rahman \\ Department of Paediatrics, Bangabandhu Sheikh Mujib Medical University, Dhaka, Bangladesh \\ Email address: \\ humayunshihan@gmail.com (M. H. Kabir),drlaila28@gmail.com (K. Laila),mujammeljewel@gmail.com (M. Haque), \\ shahana2pd@yahoo.com (S. A. Rahman) \\ ${ }^{*}$ Corresponding author
}

\section{To cite this article:}

Mohammad Humayun Kabir, Kamrul Laila, Mujammel Haque, Shahana Akhter Rahman. Drug Compliance in Children with Juvenile Idiopathic Arthritis and Reasons for Poor Compliance. American Journal of Clinical and Experimental Medicine.

Vol. 5, No. 1, 2017, pp. 15-18. doi: 10.11648/j.ajcem.20170501.14

Received: December 14, 2016; Accepted: December 27, 2016; Published: January 23, 2017

\begin{abstract}
Children with chronic disorders are at risk for a higher medication non-adherence. Juvenile idiopathic arthritis (JIA) is a chronic childhood disorder where often medication non-adherence is an issue. The purpose of this study was to assess drug compliance of JIA patients,to find out the proportion of patients having poor drug compliance and to find out possible reasons of poor drug compliance. This was a retrospective study conducted in the Pediatric Rheumatology follow up clinic, Department of Pediatrics, Bangabandhu Sheikh Mujib Medical University, Dhaka, Bangladesh. All JIA patients attending pediatric rheumatology follow up clinic during the study period was included. Data were collected using a structured questionnaire. Among the total 900 registered JIA cases, 116(12.9\%) patients had poor compliance. Among 116 poor compliant patients, systemic onset JIA cases were the highest (34.5\%), followed by enthesitis related arthritis $(30.2 \%)$. Among the poor compliant patients, $6.9 \%$ patients had regular follow up and $93.1 \%$ had irregular follow up. Major reasons of poor compliance were financial crisis, lack of awareness and difficulty in transportation. Poor compliance to treatment of JIA in our setting was common. The most important reason for poor compliance was financial crisis.
\end{abstract}

Keywords: Juvenile Idiopathic Arthritis, Poor Compliance, Follow Up

\section{Introduction}

Juvenile idiopathic arthritis (JIA) is the most common rheumatic disease in children and one of the common chronic illnesses of childhood. The worldwide incidence of JIA ranges from 0.8 to $22.6 / 100,000$ children per year, with prevalence ranges from 7 to $401 / 100,000$ [1]. The treatment of JIA in children is complex \& challenging. Medical treatment of JIA includes NSAIDs, DMARDs, Corticosteroids, Biological agents and other supportive drugs [1]. Duration of treatment of JIA is usually long and needs regular follow up and good compliance for clinical remission.

According to the World Health Organization, drug compliance can be defined as the degree of agreement between the patient's behavior-taking medication, following a diet or implementing lifestyle changes-and the recommendations from a health care provider [2]. Regarding therapy, the term "good compliance" is used when there is regular use of more than $80 \%$ of medications and other prescribed treatment modalities [3]. Several factors are there related to poor compliance in JIA and different degrees of association exists between them. To date, measuring patient medication adherence and using interventions to improve adherence are uncommon practices in routine clinical care and acute care settings [4]. Therefore, it is imperative to continue to study the reasons for medication non-adherence. The present study assessed the proportion of JIA patients having poor drug compliance and possible reasons of poor drug compliance.

\section{Materials and Methods}

It was a retrospective study conducted in Paediatric Rheumatology follow up clinic, Department of Paediatrics, 
Bangabandhu Sheikh Mujib Medical University (BSMMU), Dhaka, Bangladesh from April 2015 to March 2016. BSMMU is a tertiary care referral centre where patients are being referred from each and every parts of the country including the remote areas. The study included children with JIA who met the classification criteria set by the International League of Association for Rheumatology (ILAR) attending Paediatric Rheumatology follow up clinic, who have completed at least 1 year of treatment. A total of 1100 JIA cases were registered in the paediatric rheumatology follow up clinic. Among them 900 patients fulfilled the inclusion criteria. These 900 patients were considered as cases. Ethical clearance of the study was taken from Institutional Review Board (IRB) of BSMMU.

Hospital records of 900 JIA patients were evaluated to find out the poor compliant patients whether 1) they came for timely follow up. If not, whether they continued drugs 2) the patients who had timely follow up, whether they continued drugs regularly or not. If poor compliant, then poor compliant to which drug. 3) patients who came regularly but continued drugs like steroid, NSAID etc even after they were advised to taper off. A detailed questionnaire was completed for each poor compliant participants by interviewing them or their parents. Information were also obtained from their medical records. Investigators requested the poor compliant patients to come for a follow up over telephone. They were requested three times to attend the outpatient department for the follow up. Even then, if anyone failed to come, data were collected over telephone. Collected data included age, gender, subtypes of JIA, drug compliance, regularity of follow up and reasons for poor compliance and irregular follow up. The patient's medication details werealso recorded.

\section{Working definitions [2]}

Regular follow up-Patients who attended the outpatient department on the scheduled date or within 30 days of the scheduled date was considered as having regular follow up.

Irregular follow up-Those who did not attend the outpatient department within 30 days of the scheduled date for follow up.

Good compliance-Patients who took all drugs at the recommended dose and duration.

Poor compliance- Patients who did not take all drugs or took $50 \%$ or less of the recommended drug including dose and duration.

Non compliance- Patients who stopped one or more of the advised drugs.

Polypharmacy- Polypharmacy is the use of 4 or more medications by a patient.

\section{Results}

The study included 900 registered JIA cases. Among them $12.9 \%$ had poor compliance (Table I). Systemic onset JIA cases were the highest (4.44\%) among the cases followed by enthesitis related arthritis $(3.88 \%)$. Table-II showed the socio-demographic characteristics of the patients. Highest number of patients $(55.2 \%)$ were within the age range of 10 18 years. Mean age was $10.86 \pm 3.92$ years. Male-female ratio was 1.5 : 1 . Only $8(6.9 \%)$ patients came for regular follow up but had irregular medication and majority of the patients 108 (93.1\%) came for irregular follow up (Table III). Out of 108 patients who had irregular follow up, maximum cases $(61.1 \%)$ came after 3 months of the scheduled date, $13.9 \%$ came after 6 months and $25.0 \%$ patients came after 12 months of scheduled follow up date. NSAIDs were the most common drugs which were taken irregularly, followed by DMARDs and supporting drugs. Majority of the patients $(54.3 \%)$ had financial problem, $31.9 \%$ patients had lack of awareness. The other reasons for poor compliance were difficulty in transportation, non-availability of medicine locally, influence of traditional healers, poly-pharmacy, fear of subcutaneous injection and drug side effects like nausea and vomiting, family disharmony etc. Some patients had more than one reasons of poor compliance. So the summation of frequencies was more than $100 \%$ (Table IV).

Table 1. Frequency of JIA patients who were poor-compliant to treatment $(n=900)$.

\begin{tabular}{llll}
\hline Subtypes of JIA & Number of patients & Number of poor compliant patients & Percentage \\
\hline Systemic onset JIA & 202 & $40 / 900$ & 4.44 \\
Enthesitis related arthritis & 310 & $35 / 900$ & 3.88 \\
RF positive polyarticular JIA & 45 & $20 / 900$ & 2.22 \\
RF negative polyarticular JIA & 185 & $9 / 900$ & 1.0 \\
Oligoarticular JIA extended & 18 & $8 / 900$ & 0.88 \\
Oligoarticular JIA persistent & 140 & $4 / 900$ & 0.44 \\
Total & 900 & $116 / 900$ & 12.9 \\
\hline
\end{tabular}

Table 2. Socio-Demographic characteristics of the poor compliant JIA cases $(n=116)$.

\begin{tabular}{lll}
\hline Socio-demographic characteristics & Frequency & Percentage (\%) \\
\hline Age & & 8.6 \\
$1-5$ yrs & 10 & 36.2 \\
$5-10$ yrs & 42 & 55.2 \\
$10-18$ yrs & 64 & 100.0 \\
Total & 116 & $10.86 \pm 3.92$ \\
Mean \pm SD & $(4-17.5)$ yrs & \\
Range & & \\
Sex & \\
\hline
\end{tabular}




\begin{tabular}{lll}
\hline Socio-demographic characteristics & Frequency & Percentage (\%) \\
\hline Male & 69 & 59.5 \\
Female & 47 & 40.5 \\
Total & 116 & 100.0 \\
\hline
\end{tabular}

Table 3. Distribution of the poor compliant JIA patients by types of follow up $(n=116)$.

\begin{tabular}{lll}
\hline Type of follow up & $\mathbf{N}$ & $\mathbf{\%}$ \\
\hline Regular & 8 & 6.9 \\
Irregular & 108 & 93.1 \\
After 3 months & 66 & 61.1 \\
After 6 months & 15 & 13.9 \\
After 12 months & 27 & 25.0 \\
\hline
\end{tabular}

Table 4. Reasons of poor compliance among the cases $(n=116)$.

\begin{tabular}{lll}
\hline Reasons of poor compliance & Frequency & Percentage (\%) \\
\hline Financial crisis & 63 & 54.3 \\
Lack of awareness & 37 & 31.9 \\
Traditional healers & 18 & 15.5 \\
Polypharmacy, injection, side effects & 16 & 13.8 \\
Non-availability of medicine & 15 & 12.9 \\
Chronicity of disease & 10 & 8.6 \\
Difficulty in transportation & 8 & 6.9 \\
Familial disharmony & 7 & 6.03 \\
\hline
\end{tabular}

\section{Discussion}

Compliance to recommended treatments is of great importance in JIA. The effects of medication compliance are directly related to disease outcome, quality of life and prognosis [5]. This study was conducted among the 900 registered JIA cases fulfilling the inclusion criteria. A total of $12.9 \%$ poor compliant JIA patients were identified. In 2012, Pelajo et al conducted a similar study in two rheumatology clinics in Boston and Rio de Janeiro to see the adherence to treatment. Overall rate of non-adherence was $18 \%$ in that study. The rate of reported non-adherence was $8 \%$ in Boston and $24 \%$ in Rio de Janeiro ( $\mathrm{P}=0.012)$ [5]. Another study was conducted by Bugni et al to see compliance to treatment among all rheumatological diseases, not only JIA. In that study, a total of $47 \%$ of patients showed poor overall adherence, where $20.2 \%$ had poor medication adherence [6].

Most of the patients $(55.2 \%)$ were above 10 years of age. Mean age was $10.86 \pm 3.92$ years. Male: female ratio was 1.5 : 1. Feldman et al. conducted a study to see the perceived adherence to prescribed treatment in juvenile idiopathic arthritis, where the mean \pm SD age was $10.2 \pm 4.4$ years (range 2-18 years) which is similar to our study, but a total of $69 \%$ of the sample were female in that study [7]. A large number of patients with the diagnosis of enthesitis related arthritis in the present study might influence the mean age and male predominance. It also might be a reflection of better attention and care of male child in comparison to the female child in our perspective. In 2015, Senzaki conducted a pilot study and reported that, results of medication non-adherence did not relate to gender, ethnicity, or duration of diagnosis. However, there was a statistical relationship between age and adherence to prescribed medications. Older patients (age >
15 years) were more likely to be adherent than younger children (ages $<14$ years) [8]. The present study contradicts this finding as older patients were less adherent to medication

Among 116 poor compliant JIA patients, systemic onset JIA cases was the majority followed by enthesitis related arthritis and RF positive polyarticular JIA patients (Table I). It is an established fact that, systemic onset JIA is one of the worst subtype of JIA. It has waxing and waning course with serious systemic manifestations at times. RF positive polyarticular JIA is another subtype with severe manifestation and poor prognosis. So it is difficult to accept the fact that systemic onset JIA and RF positive polyarticular JIA patients were poor-compliant. No other similar study was available to compare the rate of compliance in different types of JIA.

Among the poor compliant JIA patients, majority of the patients $(93.1 \%)$ had irregular follow up (Table III). Regular follow up is very important for any patient with chronic illness. During each follow up, subjective and objective assessment of a patient are judged and patients and care givers are counseled for compliance to medication and next follow up plan. It is to be noted here that, in the paediatric rheumatology clinic, BSMMU, maximum patients attend from outside Dhaka. So, the best possible follow up date is provided considering disease condition, socioeconomic and academic schedule of the attendants and the patient. Even then it was found that a large number of patients had irregular follow up. It was found that $25 \%$ of the poor compliant patients had their subsequent follow up after 12 months of the scheduled date.

Medical treatment of juvenile idiopathic arthritis (JIA) has much advanced during the last decades, and improved prognosis is a reality nowin daily clinical practice. Although the medications are effective in combating disease, their full benefits are often not realized and many patients do not take their medications as prescribed. In the current study, we have seen that, as high as $12.9 \%$ patients took medication irregularly whether they had regular follow up or not. NSAIDs were mostly taken irregularly $(73 \%)$ followed by DMARDs (72\%) and supporting drugs (65\%).

In our study, reasons for poor compliance were tried to explore. Majority of the patients $(54.3 \%)$ had financial problem and $31.9 \%$ patients had lack of awareness. The other reasons for poor compliance were difficulty in transportation, non-availability of medicine in the locality, influence of traditional healers, polypharmacy, fear of subcutaneous injection, drug side effects andfamily disharmony. It is found in several studies that forgetfulness was a common reason for medication non-adherence [9]. In the present study, however, no case of forgetfulness was found.

One solution to increase medication adherence and understanding is the Teach Back Method [10]. This tool has 
been studied and utilized in the past decade in clinics and acute settings, primarily with residents. Rapoff et al. suggested the following strategies for enhancing adherence: cueing (paring medication with an established behavior such as brushing one's teeth), monitoring (using a calendar to track when medication is taken), positive reinforcement (praising and rewarding with special tokens when medication is correctly taken) and discipline (using time-out for defiant refusals to take one's medication) [11].

\section{Conclusion}

Among the total 900 registered JIA cases, 12.9 percent patients had poor drug compliance. Among them ninety three percent patients had irregular follow up and irregular medication, whereas about 7 percent patients had regular follow up but irregular medication. Common reasons of poor compliance were financial problem, lack of awareness, poor counseling, poly-pharmacy and scarcity of drugs in the locality. Adequate supply of drugs and better counseling may help in good compliance. Further study in a large scale may be helpful for better identification of poor compliance and suggestions to overcome it.

\section{References}

[1] Klein A, Kaul I, Foeldvari I, Ganser G, Urban A \& Horneff G. Efficacy and safety of oral and parenteral methotrexate therapy in children with juvenile idiopathic arthritis: An observational study with patients from the German Methotrexate Registry. Arthritis Care Res 2012; vol. 64(9), pp. 1349-1356.

[2] World Health Organization. WHO Report on Medication Adherence, Using teach-back for patient education and selfmanagement. American Nurse Today 2011; vol. 7(3), pp. 1-3.
[3] Viswanthan $\mathrm{M}$, Golin $\mathrm{CE}$ \& Jones CD. Interventions to improve adherence to self-administered medications for chronic diseases in the United States: A systematic review. Ann Intern Med 2012; vol. 157 (11), pp. 785-795.

[4] Heidenreich PA. Patient adherence: The next frontier in quality improvement. Am. J. Med 2004; vol. 1(17), pp. 130132.

[5] Pelajo CF, Sgarlat CM, Lopez-Benitez JM, Oliveira SKF, Rodrigues MCF \& Szatanjbok FR. Adherence to methotrexate in juvenile idiopathic arthritis. Rheumatol Int. 2012; vol. 32, pp. 497-500.

[6] Bugni VM, Ozaki LS, Okamoto KY, Barbosa CM, Hilario MO \& Len CA et al. Factors associated with adherence to treatment in children and adolescents with chronic rheumatic diseases. J. Pediatr 2012;vol. 88 (6), pp. 483-488.

[7] Feldman DE, De Civita M, Dobkin PL, Malleson P, Meshefedjian G \& Duffy CM. Perceived adherence to prescribed treatment in juvenile idiopathic arthritis over a oneyear period. Arthritis Rheumatol 2007; vol. 57, pp. 226-233.

[8] Senzaki, Susan Viola, "Identifying Factors in Medication Non-Adherence in Teens Diagnosed with Juvenile Arthritis: A Pilot Study"(2015). Doctoral Projects. Paper 3.

[9] Rapoff MA, Belmont J, Lindsley C, Olson N, Morris J \&Padur J. Prevention of non-adherence to non steroidal antiinflammatory medications for newly diagnosed patients with juvenile rheumatoid arthritis. Health Psychol 2002; vol. 21(6), pp. 620-623.

[10] Kessels RP. Patients' memory for medical information. J R Soc Med. May 2003;96(5):219-22.

[11] Rapoff MA. Management of adherence and chronic rheumatic disease in children and adolescents. Best Prac. Res.Clin. Rheumatol 2006; vol. 20(2), pp. 301-314. 